\title{
Fracture bone healing and biodegradation of AZ31 implant in rats
}

C Iglesias ${ }^{\mathrm{a}}$, OG Bodelón ${ }^{\mathrm{b}}$, R Montoya ${ }^{\mathrm{b}, 1}$, C Clemente $^{\mathrm{c}}$, MC Garcia-Alonso $^{\mathrm{b}}$, JC Rubio ${ }^{\mathrm{d}}$ and ML Escudero ${ }^{\mathrm{b}^{*}}$

a Department of Plastic Surgery. Hospital Universitario La Paz, Madrid, 28046, Spain.

b Department of Surface Engineering, Corrosion and Durability, National Centre for Metallurgical Research (CENIM), CSIC, Madrid, 28040, Spain.

c Department of Human Anatomy and Embryology, School of Medicine, University of Alcalá, Alcalá de Henares, Madrid, Spain.

d Department of Trauma and Orthopedic Surgery. Hospital Universitario La Paz, Madrid, 28046, Spain.

* Corresponding author: María Lorenza Escudero. Department of Surface Engineering, Corrosion and Durability, National Centre for Metallurgical Research (CENIM-CSIC), Postal address: Avd/ Gregorio del Amo nº, 28040 Madrid, Spain. Phone number: +34 91 5538900. Fax: +34 91 5347425. e-mail address: escudero@ cenim.csic.es

Keywords: AZ31 magnesium alloy, fracture healing, biodegradation, tomography, histomorphometry, in vivo.

\begin{abstract}
The ideal temporary implant should offer enough mechanical support to allow healing of the fracture and then biodegrade and be resorbed by metabolic mechanisms without causing any toxic effect. The aim of this research has been to simultaneously study in situ bone healing and the biodegradation of AZ31 $\mathrm{Mg}$ alloy as an osteosynthesis material. The in vivo study was carried out in AZ31 implants with and without $\mathrm{Mg}$ fluoride coating inserted in un-fractured and fractured femurs of Wistar rats for long experimentation time, from 1 to 13 months, by means of computed tomography, histological and histomorphometric analysis. Tomography analysis showed the bone healing and biodegradation of AZ31 implants. The fracture is healed in $100 \%$ of the animals, and AZ31 maintains its mechanical integrity throughout the healing process. Biodegradation was monitored, quantifying the evolution of gas over time by 3D composition of tomography images. In all the studied groups, gas pockets disappear with time as a result of the diffusion process through soft tissues. Histomorphometric studies reveal that after 13 months the $46.32 \%$ of AZ31 alloy has been resorbed. The resorption of the coated and uncoated AZ31 implants inserted in fractured femurs after 1,9 and 13 month has not statistically significant differences. There is a balance between the biodegradation of AZ31 and bone healing which allows the use of AZ31 to be proposed as an osteosynthesis material.
\end{abstract}

1present address: Research group Electrochemical and Surface Engineering. Vrije Universiteit Brussel. Campus Etterbeek Pleinlaan 2, 1050 Brussels 


\section{Introduction}

Metallic implants are commonly used in osteosynthesis due to their appropriate mechanical properties and biocompatibility [1]. The biodegradability and resorbability of magnesium makes it a particularly attractive material for medical use in fields such as endovascular surgery [2] and fracture osteosynthesis [3]. Magnesium has a similar Young's modulus to bone, which is an impediment to stress shielding phenomena [4], and also promotes bone formation [5]. However, Mg presents a fast biodegradation rate, which in chloride-containing physiological media is accompanied by abundant gas formation [6-7]. The evolution of gas is an important factor as it can alter bone remodelling processes.

One of the current issues in any type of fracture osteosynthesis is whether or not the implant needs to be removed once the damage has been repaired. The ideal temporary implant should provide sufficient mechanical support to allow the fracture to heal, and once this has been consolidated should then biodegrade and be resorbed and expelled by conventional metabolic mechanisms without causing any toxic effect on the organism $[8,9]$. In the last few years $\mathrm{Mg}$ and its alloys have been proposed as appropriate implant materials in preference to polymeric materials, which are also biodegradable but afford less strain resistance and poorer mechanical properties [10]. The properties of $\mathrm{Mg}$ that make it suitable for endoprosthesic replacements are its low density of $1.4 \mathrm{~g} / \mathrm{cm}^{3}$, its Young's modulus of 40-45 GPa close to that of bone (10-40 GPa), its biocompatibility [11], biodegradability, and its ability to be resorbed without causing local or systemic toxic effects $[8,9]$.

Different alternatives have been proposed to reduce the fast $\mathrm{Mg}$ corrosion rate and improve its mechanical properties, such as the development of new processes to achieve a suitable grain size [12-14], alloying with other materials [7, 15], or the performance of surface modification treatments [16-19]. In the latter case, chemical conversion treatments in hydrofluoric acid have proven to reduce the corrosion susceptibility of $\mathrm{Mg}$ and its alloys by generating an inert $\mathrm{Mg}$ fluoride layer on the metal surface [20,21]. 
The object of this research has been to simultaneously study in situ bone healing and the biodegradation of AZ31 Mg alloy as an osteosynthesis material in rat femurs. The variables studied have been the alloy's biocompatibility in healthy and broken bone, its biodegradation rate with time, and the influence of surface treatment. The conversion treatment was only studied in fractured bones, considering the real application in the clinical practice. The protection of the coating can exert a clearer influence when the biodegradation process is more favoured as it is the case of larger electrolyte volumes bathing the implant (bone fracture). This is one of the few studies in which a fracture model such as can be found in clinical practice has been performed.

\section{Materials and methods}

\subsection{Sample preparation}

The AZ31 alloy was received from Magnesium Elektron Ltd. in the form of a rolled $3 \mathrm{~mm}$ thick sheet in O-temper condition (annealed at $345^{\circ} \mathrm{C}$ ). The chemical composition of the AZ31 alloy was determined by wavelength dispersion X-ray fluorescence (WDXRF) to be: $3.37 \pm 0.09$ wt.\% Al, $0.78 \pm 0.04$ wt. $\% \mathrm{Zn}, 0.22 \pm 0.01$ wt.\% $\mathrm{Mn}$ (balance $\mathrm{Mg}$ ). AZ31 was machined to form cylinders of $20 \mathrm{~mm}$ in length and $1 \mathrm{~mm}$ in diameter, with an average weight of $28.0 \pm 0.3$ mg. The cylindrical specimens were rounded at both ends in order to eliminate sharp edges, to facilitate their insertion in the laboratory animals, and to avoid edge effects on the degradation rate. Prior to their insertion the surface of the pins was ground with $\mathrm{SiC}$ abrasive papers and finally polished with $1 \mu \mathrm{m}$ diamond suspension. Finally, the AZ31 pins were rinsed ultrasonically in ethanol.

To reduce the biodegradation rate of AZ31 implants, half of the pins were subjected to a chemical conversion treatment applied by immersion of the pins in 48 wt.\% HF solution at room temperature for 24 hours, followed by rinsing with deionised water and drying in a stream of warm air. This treatment gave rise to the magnesium fluoride $\left(\mathrm{MgF}_{2}\right)$ protective layer [20]. 
Samples were gamma-ray sterilised prior implantation.

\subsection{Surgery}

36 female Wistar rats of three months of age and approximately $200 \mathrm{~g}$ body weight (bw) were used. The animals were treated in accordance with European Union Guidelines for Ethical Care of Animals (86/609CEE) and Spanish regulations (RD 1201/2005).

The rats were randomly divided into four different groups of nine animals each. All the animals belonging to Groups 1 to 4 underwent surgical implantation of one single AZ31 alloy pin in its left hind leg. The animals in Groups 1 and 2 (no fractured femur) were fitted with an intramedullary pin (see protocol 1 below), while the animals belonging to Groups 3 and 4 (with fractured femur) underwent intramedullary pinning (see protocol 2 below). The pins implanted in groups 1 and 3 were previously subjected to surface modification with HF treatment, while the pins implanted in groups 2 and 4 remained untreated.

The devices were implanted in the femur under general anaesthesia following two different protocols:

\section{Protocol 1. Intramedullary pinning no fractured femur}

The rat was placed in supine position to facilitate an anteromedial approach to the knee. A 1.1 mm hole was drilled with a surgical punch through the intercondylar notch into the femoral intramedullary space. The implant was then inserted into the intramedullary channel by retrograde insertion.

\section{Protocol 2. Intramedullary pinning fractured femur}

In a lateral approach to the rat's femur, a comminuted middle shaft fracture was made about 10 $\mathrm{mm}$ from the joint line. To reproduce the physiological conditions of the fracture, a unicortical cut was made with a Gillies saw followed by manual breakage of the other cortex, adopting a comminute long oblique fracture pattern. The endomedullary pin was inserted into the distal 
medullary channel and the fracture was reduced by threading the proximal bone fragment with the pin.

Following the operation, each rat received a prophylactic dose of analgesics and antibiotics: 5 mg Enrofloxacin (BAYTRIL ${ }^{\circledR}, 10 \mathrm{mg} / \mathrm{kg}$ ) and Meloxicam $\left(\right.$ METACAM $^{\circledR}, 2 \mathrm{mg} / \mathrm{kg}$ ), both in a single dose via subcutaneous injection. All the animals were kept in the same conditions.

\subsection{Computed Tomography studies}

Computed Tomography, (CT) is taken as a suitable image diagnosis method in view of its high resolution to evaluate the biodegradation of the material and to monitor bone healing [22]. Tomography offers an easy-to-interpret image that allows simple identification of the material's position and outline and the morphology of the bone. It also permits analysis of the volume of gas (from the dark shades present on the images) that is released as a result of the electrochemical biodegradation reaction:

$\mathrm{Mg}+2 \mathrm{H}_{2} \mathrm{O} \rightarrow \mathrm{Mg}^{2+}+2 \mathrm{OH}^{-}+\mathrm{H}_{2}(\mathrm{~g})$

The pins implanted in the femurs were analysed in situ on a weekly basis by means of tomography in the rats euthanized after one month and on monthly bases in those euthanized after 9 and 13 months. To assure their immobilisation during scanning, the rats were anesthetised with $2 \%$ isofluorane and then placed on the scanner bed (Albira ARS PET/CT hybrid tomograph, Oncovision, Valencia, Spain). The parameters chosen for the performance of scanning were as follows: 600 projections per acquisition, a radiation intensity of $0.8 \mathrm{~mA}$ and a voltage of $45 \mathrm{kV}$. Prior to the tomography study a scout image was taken to assure the right position of the femur in the field of view $(64 \times 64 \mathrm{~mm})$ of the scanner. After this, the tomography images were reconstructed using a filtered back projection algorithm. The final tridimensional images have a voxel size of $0.125 \mathrm{~mm}^{3}(512 \times 512 \times 512$ pixels $)$ and were saved in an analytical format for subsequent analysis. 
The volumes were interactively segmented using ITK-SAP [23]. Each volume was segmented into gas and implant, and the volume of each structure was computed using the resolution information present in the DICOM header.

\subsection{Histological and histomorphometric analysis}

Upon completion of the scheduled monitoring times of 1,9 and 13 months, the rats were euthanized by intraperitoneal injection of $0.4 \mathrm{mg}$ sodium pentobarbital (Dolethal ${ }^{\circledR}$ ) diluted in serum.

The tissue response around the AZ31 implants was assessed by means of histological and histomorphometric analysis. The femurs with implants were extracted and fixed in $10 \%$ buffered $\mathrm{pH} 7$ formaldehyde and dehydrated in grading hydroxylethylmetacrylate resin concentrations, as mentioned in Donath and Breuner's method [24]. The femurs were cut into blocks and then set in Technovit ${ }^{\circledR}$ resin. The cutting and grinding of hard tissues was performed with an EXAKT sawing machine and grinding equipment (Leica Microsystems, Wetzlar, Germany). Five cross sections of about $50 \mu \mathrm{m}$ thickness were obtained from each femur. Usual histological laboratory stains were used: toluidine blue with Weigert haematoxylin (Merck) and Masson's trichrome (Merck). Also a variation of blue toluidine (Merck) staining, which does not require prior removal of the resin, has been used. Samples have been stained with Weigert haematoxylin, washed in water and immersed in blue toluidine at $\mathrm{pH} 4$. The prior use of haematoxylin enhances the blue toluidine effect, and cell nuclei appear very dark, almost black. The intercellular substance is clearly differentiated in several shades of blue, depending on the degree of maturity of the bone. The Masson's trichrome makes it possible to appreciate the uncalcified osteoid tissue (orange), the calcified mature bone tissue (green) and the cell nuclei (dark red).

The stained cross sections of femurs with implants were studied with an optical microscope (Zeiss). The images were transferred to MIP 4 software (Digital Image Systems, SL, Barcelona) to carry out the histomorphometric analysis. This software identifies the initial area occupied by 
the implant which is easily recognised by the circle of new tissue around it and highlights the remaining AZ31 alloy implant by colour density so the loss of implant in each section can be calculated.

\subsection{Statistics}

The data obtained in tomography experimentation were summarised as mean \pm standard deviation (S.D.). T-tests or Mann-Whitney tests were used to compare two independent groups. ANOVA for repeated measures and pairwise comparison with Bonferroni correction were used to compare means at different time-points. $p$-Values $\leq 0.05$ were considered as statistically significant. Statistical analysis was performed with Statgraphics Online.

\section{Results}

\subsection{Effect of the femur fracture on the biodegradation rate}

\subsubsection{Computed tomography}

Fig. 1 shows representative tomography images after 1, 3, 5, 9 and 13 months for groups 3 and 4 with fractured femur (Figs. 1a-e) and groups 1 and 2 with un-fractured femur (Figs. 1f-j), respectively.

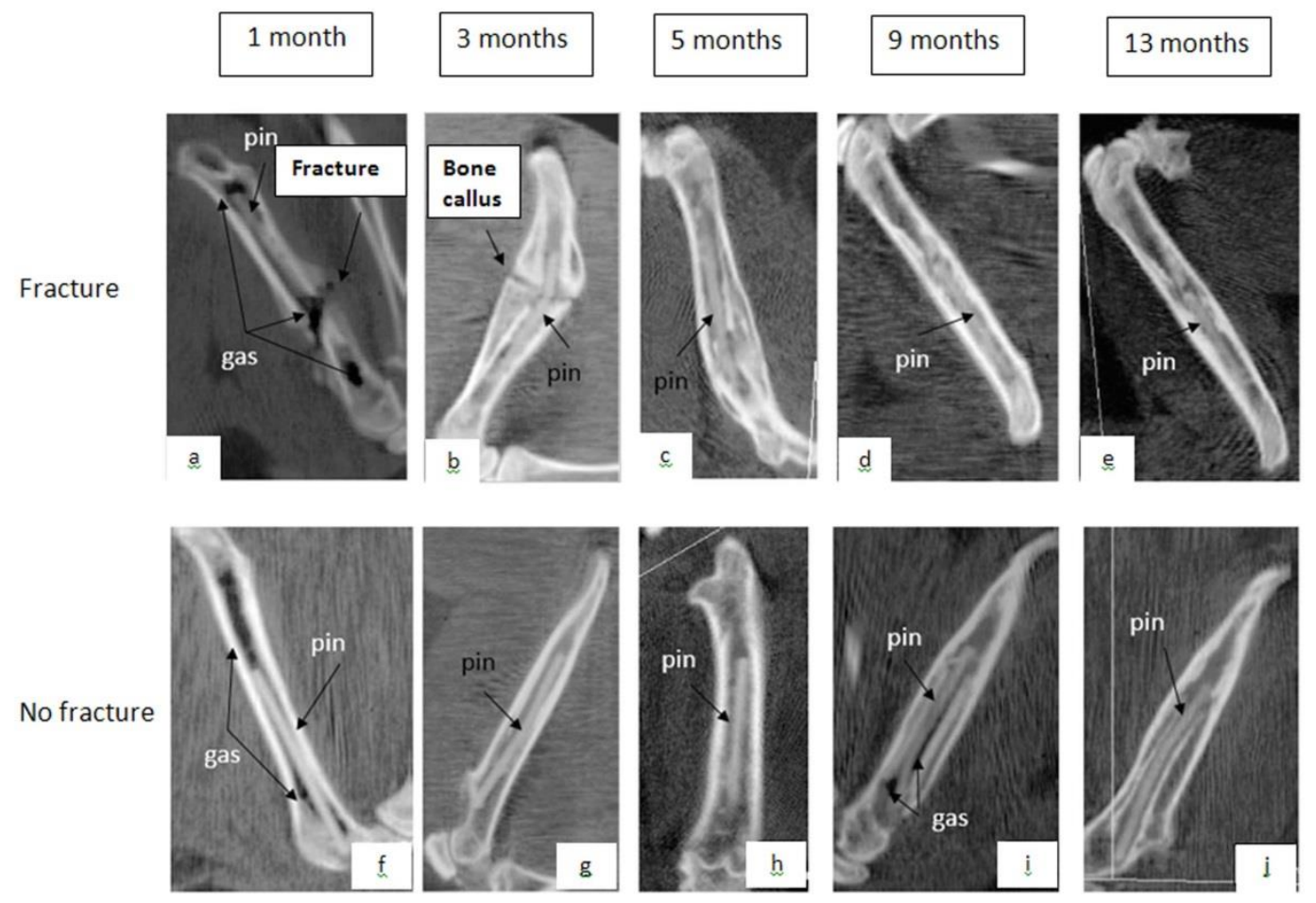


Figure 1. Computed tomography images of implanted AZ31 magnesium alloy after 1, 3, 5, 9 and 13 months of evolution in Wistar rats, for groups 2 and 4, uncoated implants. From a- e) with fractured femurs, and from $\mathrm{f}-\mathrm{j}$ ) with un-fractured femurs.

These images show the changes experienced by the implant, the gas produced as a consequence of its degradation, and the evolution of the bone between 1 and 13 months after implantation. Observation of the implants shows a well-defined outline in the images for 1 month in unfractured femurs (Fig. 1f) which continues up to the third month (Fig. 1g). From this implantation time, the outline gradually becomes diffuse and is more difficult to be delimited. With regard to gas pockets, the maximum volume of gas is observed after 1 month of implantation (Figures 1a and 1f). In the group of rats with fractured femur (groups 3 and 4) gas is less abundant and is mainly located at the fracture site and extending beyond its limits into the surrounding soft tissues, and it is also located at the metaphysis (Fig. 1a). From the third month of implantation (Fig. 1b) the amount of gas decreases significantly, with only some bubbles remaining in the vicinity of the fracture site. In the subsequent implantation times the gas has almost completely disappeared. In the groups of rats of un-fractured femurs (groups 1 and 2), a large amount of gas appears after one month of implantation located at the metaphysis and around the implant (Fig. 1f). This gas is barely perceptible after five months (Fig. 1h), but reappears in the ninth month (Fig. 1i) before finally disappearing completely (Fig. 1j).

In the rats with fractured femur, bone healing occurred in $100 \%$ of animals and the bone callus appeared in the third month (Fig. 1b), being the fracture completely healed by the fifth month (Fig. 1c). There were no cases of non-union. The bone remodelling capacity is high, as can be seen in the tomography images for 9 and 13 months of implantation (Fig.1d and 1e), where there is no difference between the rat femurs with previous fracture and those without fracture.

Table 1 shows the quantitative evolution of the gas volume of uncoated AZ31 alloy for rats with fractured femur and un-fractured femur measured from the overlapping of tomography images. 


\begin{tabular}{|l|c|c|}
\hline $\begin{array}{c}\text { Time } \\
\text { (months) }\end{array}$ & $\begin{array}{c}\text { Fractured Femur } \\
\text { Gas volume }\left(\mathrm{mm}^{3}\right) \\
\text { Mean } \pm \mathrm{SD}\end{array}$ & $\begin{array}{c}\text { Un-fractured } \\
\text { Femur } \\
\text { Gas volume }\left(\mathrm{mm}^{3}\right) \\
\text { Mean } \pm \mathrm{SD}\end{array}$ \\
\hline $\mathbf{1}$ & $12.11 \pm 6.43$ & $19.09 \pm 8.21$ \\
\hline $\mathbf{3}$ & $6.75 \pm 3.91$ & $17.77 \pm 8.80$ \\
\hline $\mathbf{4}$ & $1.32 \pm 0.68$ & $6.02 \pm 1.07$ \\
\hline $\mathbf{8}$ & $1.29 \pm 1.24$ & $4.31 \pm 4.59$ \\
\hline $\mathbf{9}$ & $1.07 \pm 1.20$ & $3.18 \pm 4.26$ \\
\hline $\mathbf{1 0}$ & $0.96 \pm 1.21$ & $4.92 \pm 4.90$ \\
\hline $\mathbf{1 2}$ & $0.23 \pm 0.42$ & $1.56 \pm 1.07$ \\
\hline $\mathbf{1 3}$ & $0.00 \pm 0.00$ & $0.21 \pm 0.20$ \\
\hline
\end{tabular}

Table 1. Evolution of gas volume released from the biodegradation of uncoated AZ31 alloy implant versus implantation time in Wistar rats with and without fractured femur.

The volume of gas released is equivalent to the amount of AZ31 biodegraded (in mols). As can be seen in table 1, in the group of un-fractured femurs the gas accumulated after one month averages $19.09 \pm 8.21 \mathrm{~mm}^{3}$; while in the rats with fractured femur the value is lower, $12.11 \pm$ $6.43 \mathrm{~mm}^{3}$. Between 8 and 10 months of implantation the amount of gas raises in the group of animals with un-fractured femurs. The value of $1.32 \pm 0.68 \mathrm{~mm}^{3}$ is reached in the fourth month in the rats with fractured femurs, whereas in the animals with un-fractured femurs, this value is reached after 12 months $\left(1.56 \pm 1.07 \mathrm{~mm}^{3}\right)$. There were not statistically significant differences between fractured and un-fractured femur.

\subsubsection{Histological and histomorphometric analysis}

Fig. 2 shows the representative cross section images at different magnifications of the partially resorbed AZ31 implants of group 2 (un-fractured femur and without MgF2 coating) after 1 and 13 months implantation times. 

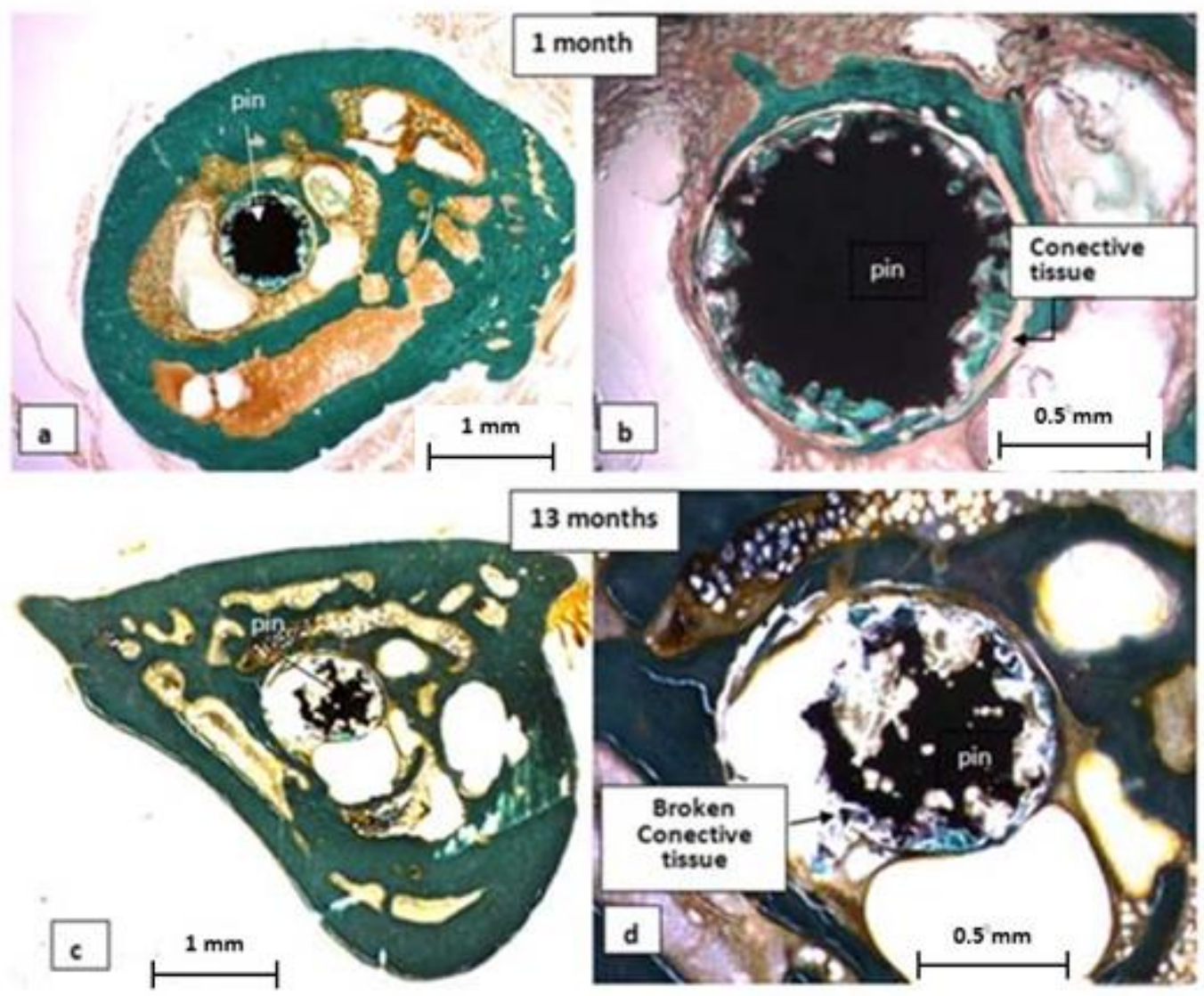

Figure 2. Histological cross-section images of the partially resorbed AZ31 magnesium alloy implants after 1 month (a, b) and 13 months (c, d). Samples a), b) and c) were stained with Masson's trichrome: green-calcified mature bone tissue; orange-uncalcified osteoid tissue; dark red-cell nuclei. Sample d) was stained with haematoxylin enhancing the blue toluidine: dark, almost black-cell nuclei; shades of blue, depending on the degree of maturity of the boneintercellular substance.

Cells and intercellular substance are differentiated depending on the staining used. However, implanted material always appears undyed and black, which are easily identified. Implants are easily recognized in the initial site of implantation by the new circle of tissue around (as shown in Figure 2). Resorbed implant appears as grey colour so the remaining area in black colour is measured.

After 1 month (Fig. 2a and b) it is possible to see a circular black area identified as the AZ31 implant and evidence of bone neoformation in the medullary cavity, distinguishing a thick trabecula of cortical bone. In the bone trabecula cavities it is possible to see bone marrow of an orange coloured dotted appearance. It can also be seen that the implant is partially resorbed at 
the edges (Fig. 2b). The resorbed area has been occupied by inorganic compounds, Mg oxides and hydroxides, corrosion products that surround the surface of the implant. On the other hand, a lamina of fibrous tissue is seen separating the original area of bone tissue that surrounds it, although no foreign body or inflammatory cells have been found. After 13 months the implant is partially resorbed (Fig. 2c and d), being possible to observe breakage of the fibrous tissue encapsulation that surrounds the biomaterial.

Histomorphometric analysis allows us to quantify the real resorption of the implant from the remaining implant areas measured on the different cross sections. The information obtained from micro CT and histomorphometric analysis is complementary and do not have necessarily to follow the same trend.

Fig. 3 quantifies the implant resorption percentages, which after 1, 9 and 13 months for the groups 2 and 4 (uncoated implants) rats with un-fractured and fractured femur, respectively. Resorption percentage was calculated considering that the resorbed implant appears as grey colour and the implant area not resorbed appears as black colour. The resorption percentage was calculated from the difference between the original volume and that remaining.

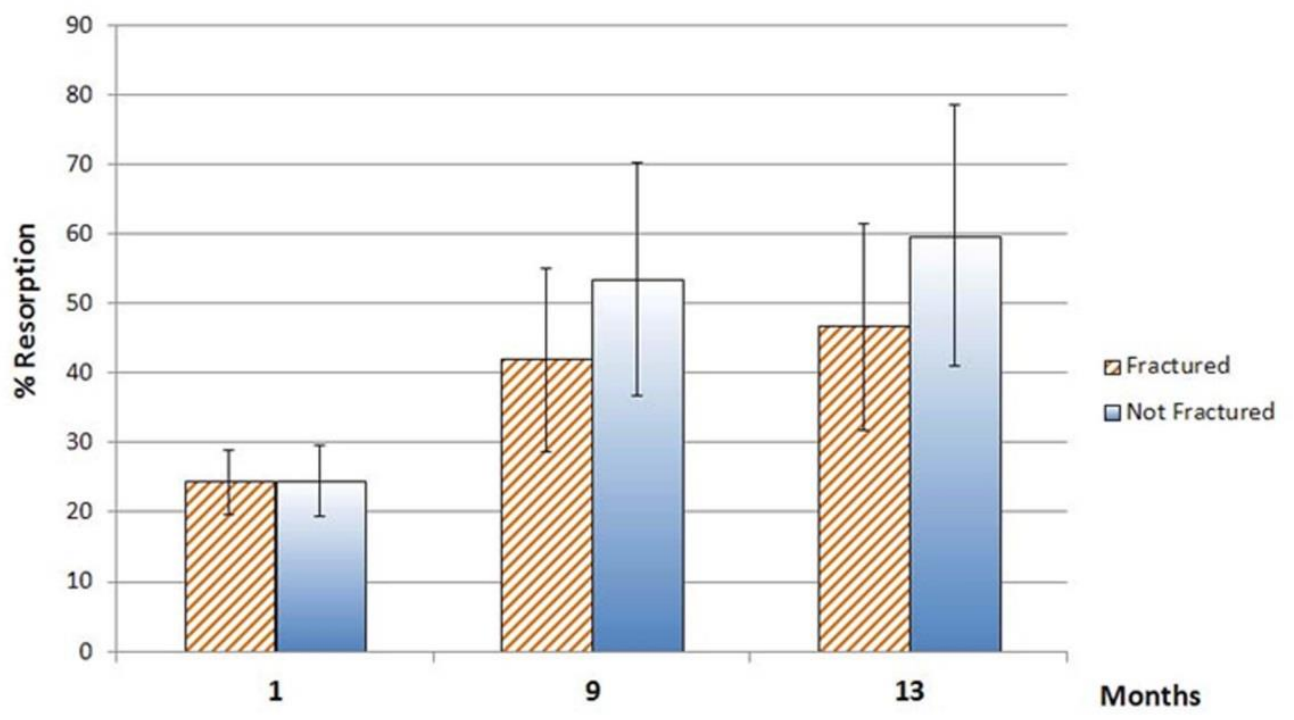

Figure 3. Percentage of AZ31 uncoated implant resorption after 1, 9 and 13 months in Wistar rats with fractured and un-fractured femurs. 
Histomorphometric studies reveal that after one month the percentage of resorbed material is similar in both groups. The corrosion that occurs does not alter its central structure and takes the form of pitting corrosion that starts on its surface. Nevertheless, resorption percentage at long implantation times is higher in the group of rats 2 without fractured femur. After 9 months, when the fractures have been consolidated, $41.85 \%$ of the material has been resorbed and its central structure is conserved.

\subsubsection{Effect of $\mathrm{MgF}_{2}$ coating on the biodegradation rate}

The analysis of the effect of $\mathrm{MgF}_{2}$ coating on the biodegradation rate of AZ31 implants, was studied in the groups 3 and 4, rats with fractured femur. Images acquired from computed tomography show the corroded implant without possible distinction between the remaining implant and the corrosion products that has been defined as the total volume of the implant. In Figure 4, the total volume of the implant including corrosion products and gas volume in AZ31 with and without $\mathrm{MgF}_{2}$ coating from 1 to 13 months in Wistar rats with fractured femurs is shown. 


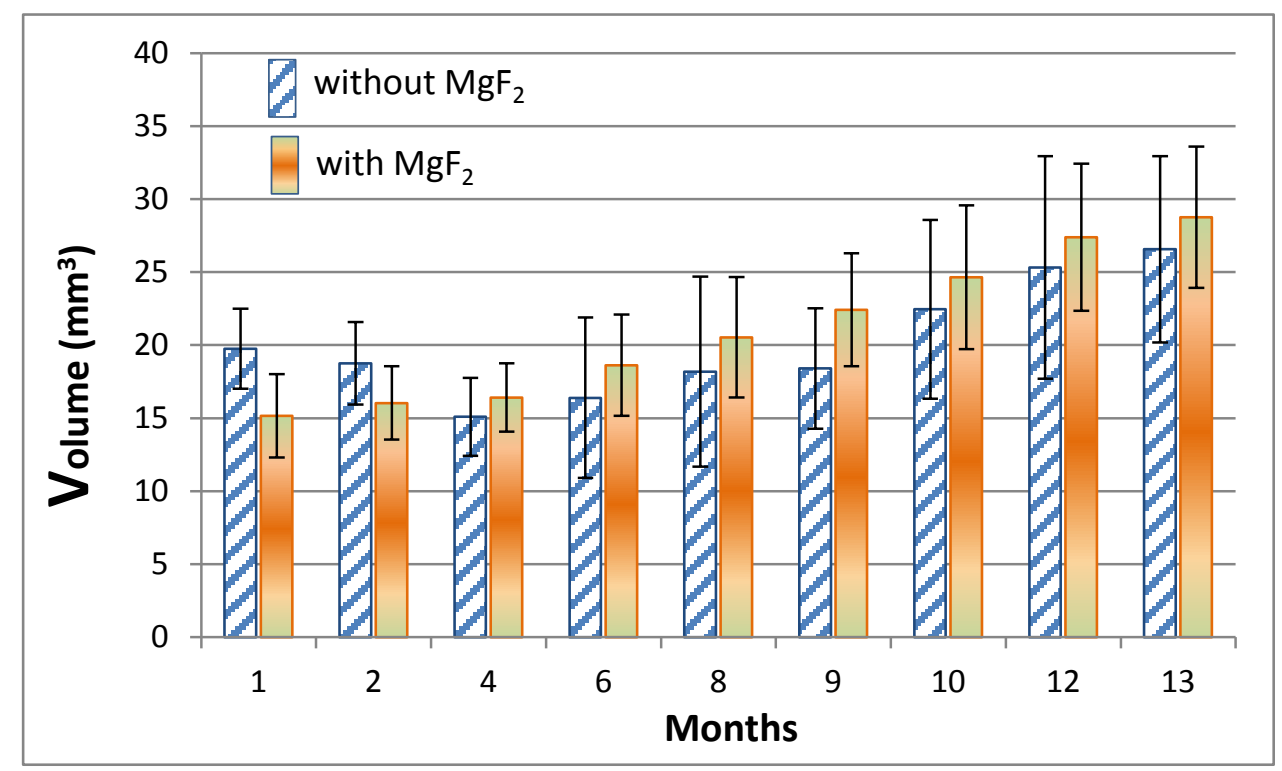

a)

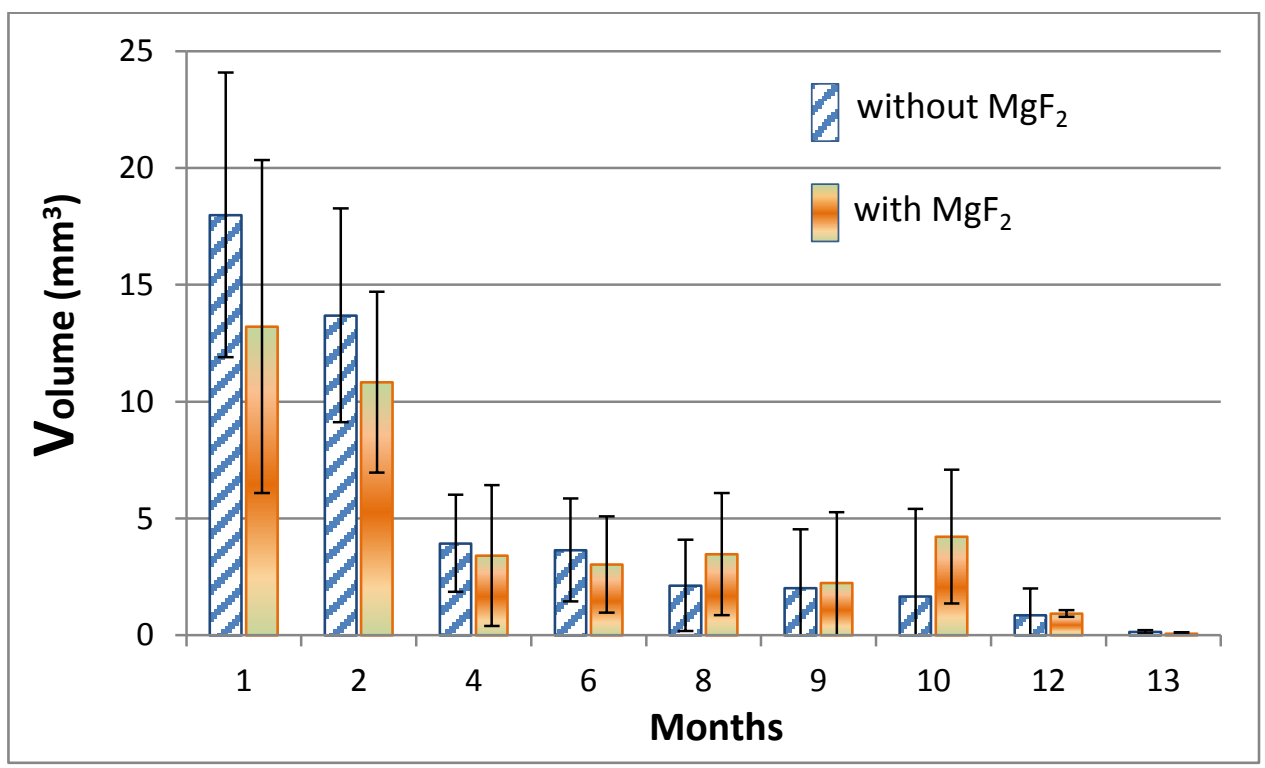

b)

Figure 4. Total volume of the implant and corrosion products, a), and gas volume, b), in AZ31 with and without $\mathrm{MgF}_{2}$ coating from 1 to 13 months in Wistar rats with fractured femur.

$\mathrm{MgF}_{2}$ coating seems to have a small influence on the biocorrosion process. The first four months of implantation, the modification of the surface slightly reduces the biocorrosion of the implant. However, from the fourth month to the end of the in vivo experimentation the trend is reversed due to a higher amount of corrosion products which increases the total volume of the implant. The evolution of the gas volume over time in coated and uncoated AZ31 implants for 
rats with fractured femurs was nearly parallel with small differences between them. There were not statistically significant differences between uncoated and coated implants.

Table 2 shows in statistical quantitative terms the percentage of biodegraded AZ31 implants with and without $\mathrm{MgF}_{2}$ coating in Wistar rats with fractured femur after 1, 9 and 13 months of implantation.

\begin{tabular}{|l|l|l|l|l|}
\hline \multicolumn{5}{|c|}{ AZ31 implant resorption (\%) } \\
\hline \multirow{2}{*}{$\begin{array}{l}\text { Time } \\
(\text { months) }\end{array}$} & MgF $_{2}$ coated implants & \multicolumn{2}{l|}{ Uncoated implants } \\
\cline { 2 - 5 } & Mean & S.D. & Mean & S.D. \\
\hline $\mathbf{1}$ & 24.52 & 5.00 & 26.34 & 5.31 \\
\hline $\mathbf{9}$ & 43.94 & 7.48 & 41.85 & 12.71 \\
\hline $\mathbf{1 3}$ & 53.51 & 16.82 & 46.32 & 15.30 \\
\hline
\end{tabular}

Table 2. Percentage of AZ31 implant resorption, after 1, 9 and 13 months with and without $\mathrm{MgF}_{2}$ coating in Wistar rats with fractured femur. S.D: Standard Deviation.

It can be seen that the resorption of the material after 1,9 and 13 month is not statistically significant between the group 3 with $\mathrm{MgF}_{2}$ coated AZ31 implant and the group 4 with uncoated AZ31 implants.

\section{Discussion}

A material that is to be used in fracture osteosynthesis must fulfil a number of basic requirements: 1) retain its mechanical properties long enough to achieve bone healing, i.e. present little degradation between weeks 3 and 16, when the bone callus is forming [25,26]; 2) be biocompatible; and 3) allow bone regeneration ad integrum (occurs between 12 and 15 months) [27-30], which means that during all this time the material must degrade at a controlled rate. The final result is an appropriate balance between the bone healing process and biodegradation of the material. 
Previous in vitro and in vivo studies have shown that $\mathrm{Mg}$ and its alloys are biocompatible materials that do not cause systemic inflammatory reactions or alterations in analytical parameters [31-33]. However, the biodegradation process of $\mathrm{Mg}$ base temporary materials in vivo presents two fundamental problems for use in clinical practice: on the one hand the fast corrosion of the material [34-36], which leads to a loss of mechanical properties and, on the other hand, the formation of gas associated with the biodegradation process, which can affect bone healing $[32,33,37]$.

4.1. Does the AZ31 corrosion rate allow the implant material to maintain its mechanical support long enough for bone healing?

The in vivo corrosion reaction takes place according to two semi-reactions:

- Anodic or oxidation: $\mathrm{Mg}^{0} \rightarrow \mathrm{Mg}^{2+}+2 \mathrm{e}^{-}$

- Cathodic or reduction: $2 \mathrm{H}_{2} \mathrm{O}+2 \mathrm{e}^{-} \rightarrow+2 \mathrm{OH}^{-}+\mathrm{H}_{2}(\mathrm{~g})$

The first semi-reaction starts on the implant surface (with or without surface treatment) right from the initial moments of its insertion. It occurs because the surface is bathed in the physiological fluid containing water and mineral salts, giving rise to the formation of $\mathrm{Mg}$ oxide and hydroxide, and probably also the phosphates and carbonates that are deposited on the metal surface as detected in in vitro and in vivo test $[35,36]$. The Mg hydroxides form a protective layer, but in a chloride medium, when the chloride ion concentration reaches $30 \mathrm{mmol} / \mathrm{L}, \mathrm{Mg}$ hydroxides are transformed into highly soluble magnesium chlorides [38]. This promotes the appearance of pitting on the implant surface. If the chloride concentration in the extracellular fluid reaches $150 \mathrm{mmol} / \mathrm{L}$, pitting corrosion becomes severe [38]. Figure 5 shows the deposited corrosion products (identified with letter b in Figure 5). 


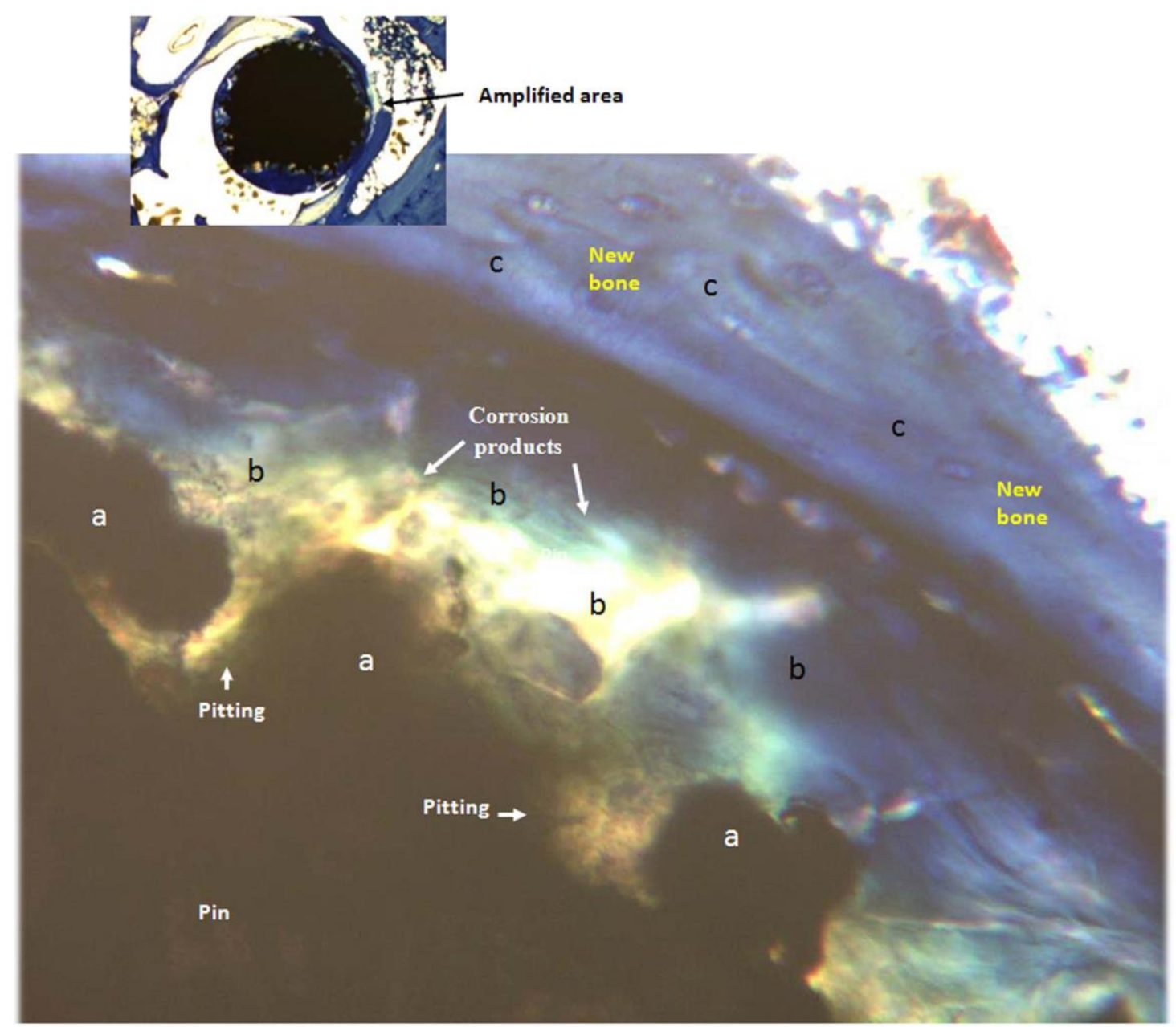

Figure 5. Histological cross-section images of implanted AZ31 magnesium alloy after 9 months in Wistar rats. Letters in the images: a-pitting corrosion b-corrosion products and c-new bone.

These Mg oxides and hydroxides promote the accumulation of calcium phosphate and therefore the formation of new bone around the implant (Figure 5c) [34]. The accumulated oxides and hydroxides have a similar atomic number to $\mathrm{Mg}$, and for this reason cannot be distinguished from the pin in computed tomography. As a result it was decided to quantify the biodegradation process by measuring the cathodic or reduction reaction (3), i.e. gas pockets by processing the images taken from tomography. The organism is able to absorb the gas produced and expel it by diffusion, but if the hydrogen formation rate is very high the gas excess is accumulated as bubbles around the implant or fracture site (Figure 1a) [35,37]. In groups 1 and 2 (un-fractured femur) the corrosion products from the biodegradation process (equation (1)) remain on the implant surface, to a certain extent protecting the surface from the corrosion process. As the 
corrosion products become more voluminous and porous, oxygen and water have access to the metallic surface, reactivating the corrosion process. For this reason gas reappears in the metaphysis in the un-fractured femur of the rats, whereas this oscillation does not take place in the rats with fractured femur (Table 1). It can be seen that gas production is much greater in the first month and subsequently drops until it almost disappears. However, in the rats with fractured femur less gas is quantified than in those un-fractured femur, and the oscillation in gas production does not occur. All of these differences may be attributed to the fact that only intramedullary gas and peri-fracture gas appears in the image quantification. The gas diffused through the soft tissues in close contact with the material is not measured, i.e. it is formed but not in enough amount to be accumulated in pockets. In other words, in a richly vascularised environment gas does not accumulate but dissolves and is eliminated by normal biological procedures [39,40]. The gas is not only $\mathrm{H}_{2}$ as appears in equations (1) and (3) [41]. As early as 1900, Payr [42] hypothesised that the accumulated gas was a mixture of oxygen and hydrogen. Subsequently McBride shows that there was carbon dioxide (5.6\%), oxygen (6.5\%), hydrogen (7.3\%) and nitrogen (80.6\%) [43]. Recent studies indicate not only the presence of hydrogen gas but a mixture of gases $[39,44]$.

Some authors discuss whether gas formation at the fracture site may delay or alter callus formation and therefore fracture healing [45-48]. Kraus [5] argues that callus formation in the medial cortex is greater due to the accumulation of gas in this cortex which is the area of least resistance. However, in a relatively stable synthesised femur fracture the fracture callus will always be hypertrophic and will always be greater in the medial cortex, because it supports compression forces while the outer cortex supports tension forces. According to Roux-Wolff's law [49], bone formation is guided by mechanical forces and bone has the capacity to adapt its architecture to external loads. More recently, a mathematical simulation carried out on the bone structure and its biological adaptation to the mechanical load to which it is subjected, demonstrated and validated Wolff's studies by microCT and microFEA (finite element analysis)[50]. In the present study, pseudoarthrosis does not occur in any case and the shape of 
the bone callus is homogenous for all the groups. That is why there are not clinical secondary reactions due to the gas.

It can be concluded that bone healing occurred in $100 \%$ of the study animals. The bone callus was seen in the third month (Fig. 1b) and the fracture was considered to be completely consolidated in the fifth month (Fig. 1c).

\subsection{Biocompatibility of AZ31 alloy}

Throughout the implantation time (1 to 13 months) of the AZ31 implant, coated and uncoated, the implanted material did not cause clinical alterations such as reddening or the appearance of obvious gas bubbles or deaths due to embolism. The animals subjected to a fracture of the femur could not be immobilised and showed reduced mobility for the first 3 weeks after surgery, but their habitual behaviour was not altered.

In the histological studies after 1 month of implantation it has been seen that there is neither reaction to the foreign body nor any inflammatory reaction (Figs. $2 \mathrm{a}$ and $2 \mathrm{~b}$ ). The insertion of any material in an intramedullary cavity generally leads to a process of relative osteolysis or bone resorption which is considered normal. With the AZ31 alloy no such osteolysis has taken place (Figs. 2c and 2d) and the images show osteoblast activity by the presence of bone formation. Osteoclasts were not appreciated. After this first month a thin fibrous layer is seen surrounding the material, and based on polarised light studies this layer corresponds to bone tissue immature (Fig. 2b). After 13 months the studies confirm that the material is completely surrounded by a layer of mature bone tissue. This new bone is firmly adhered to the implant surface. This represents a direct index of the implant's good biocompatibility and its osteoproliferative capacity. These results are in accordance with those obtained by other authors [21,38,51-53]. No histological differences are seen between the reactions that take place on $\mathrm{MgF}_{2}$ coated and uncoated surfaces.

Histomorphometric studies reveal that after 9 months, $41.85 \%$ of the material has been resorbed. This resorption of more than $40 \%$ after 9 months means that this material cannot be 
described as suitable for the osteosynthesis of bones subjected to great loads, due to the possible loss of mechanical properties [54].

\subsection{Effect of $\mathrm{MgF}_{2}$ on the biodegradation rate of AZ31 implants}

The magnesium fluoride coating, which in in vitro studies was seen to be highly efficient at reducing the corrosion rate of AZ31 alloy $[55,56]$, has not reduced corrosion of the implant at long implantation times in the present study. Although the initial biodegradation (1 month) is similar in the implants with and without surface treatment, the biodegradation rate after 13 months is greater in the case of the implants with a magnesium fluoride coating (Table 2). The volume of corroded implants of coated AZ31 implants follow the same trend as Thoman's studies about in vivo degradation of $\mathrm{MgCa} 0.8$ implants [57], who found that "after 6 months the volume of the coated implants was $13 \%$ higher than the volume of the uncoated pins". In general, the protective coating is compact and uniform, however over time physiological fluids pass through defects such as pores and cracks until arriving AZ31 surface, promoting nuclei for corrosion attack. Gu describes this mechanism in AZ31 alloy in vitro [36]. According to Wang corrosion accelerates if the coating is locally destroyed [7]. Studies performed with Scanning Kelvin Probe revealed [58] that the difference of potential established between areas protected by the coated surface $(790 \mathrm{mV})$ and uncoated areas $(360 \mathrm{mV})$ gives rise to galvanic corrosion couples which accelerate corrosion. It should also be remembered that the $\mathrm{pH}$ in areas adjacent to where corrosion is taking place can reach values of 9 , and at these $\mathrm{pH}$ values the magnesium fluoride coating is not stable and is easy to dissolve.

Once again the widely debated question arises as to how far the in vivo situation of magnesium is reproduced in vitro tests, given that there are many physiological factors in vivo that make in vitro studies a poor comparison [32]. In vitro studies cannot take into account variables such as electrochemical plugging by proteins, diffusion through the membranes formed by the implant, or localised $\mathrm{pH}$ alterations [59]. A variable that has been little studied is the thickness of the electrolyte bathing the implant. Montoya et al. conclude that biodegradation differs according to 
the electrolyte thickness at the implant site [60]. These authors identify by means of a mathematical model the variables that affect the biodegradation of magnesium such as the type of material (AZ31, cast $\mathrm{Mg}$ and powder metallurgical $\mathrm{Mg}$ ) thickness of the electrolyte surrounding the implant, and density of the particles embedded in it.

The surface modification of AZ31 with the magnesium fluoride coating is not justified in in vivo experimentation, so it would not be necessary to apply it to protect the implant and this would also avoid the potential problems related with fluoride ions.

\subsection{Biodegradation and bone restitution}

Bone regeneration ad integrum means that throughout the time the material presents degradation at a controlled rate until its complete disappearance.

In this study complete degradation of the material was not reached, and se its degradation kinetics beyond 13 months is not known. With regard to complete bone restitution, the computed tomography images (Figs. 1i and $1 \mathrm{j}$ ) show the bone remodelling capacity which allowed the fractured femurs to have a similar morphology to un-fractured femurs after 9 to 13 months. The balance that is sought between bone healing and biodegradation of the material seems to be achieved without difficulty for the studied group of rats with AZ31 alloy [2, 61].

In summary this animal experimentation study has shown that AZ31 alloy has real potential to be used as an osteosynthesis material in fractures. It offers the main advantage of its degradation once the healing process has finished, thus avoiding the need to remove the material.

\section{Conclusions}

- The fracture is healed in $100 \%$ of the animals, and AZ31 alloy maintains its mechanical integrity throughout the healing process. The gas produced in the biodegradation process of 
AZ31 is exchanged and dissipates quickly, not altering the formation or the morphology of the bone callus.

- AZ31 alloy is a biocompatible material with osteoproliferative properties. Surface modification with $\mathrm{MgF}_{2}$ is also well tolerated by the organism.

- There is not statistically significant differences in the resorption process between coated and uncoated AZ31 implants inserted in fractured femurs after 1,9 and 13 month.

- There is a balance between the biodegradation of AZ31 and bone healing which allows the use of AZ31 to be proposed as an osteosynthesis material, although its resorption seems to make it more advisable for bones not subjected to mechanical loads.

\section{Acknowledgements}

The authors are grateful to MINECO, Spain, for financial support of projects MAT 2008-06719C03-01-03 and MAT 2011-29152-C02-01. The authors would like to thank the technical assistance to R. Fernandez, M. Delgado, and L. Garcia from Instituto Pluridisciplinar (U. Complutense of Madrid). Also to thank N. Malpica from U. Rey Juan Carlos of Madrid for the support in the ITK-SNAP application.

\section{References}

[1] C. Hampp, N. Angrisani, J. Reifenrath, D. Bormann, J.-M. Seitz, A. MeyerLindenberg, Evaluation of the biocompatibility of two magnesium alloys as degradable implant materials in comparison to titanium as non-resorbable material in the rabbit, Materials Science \& Engineering C-Materials for Biological Applications, 33 (2013) 317-326.

[2] H. Windhagen, K. Radtke, A. Weizbauer, J. Diekmann, Y. Noll, U. Kreimeyer, R. Schavan, C. Stukenborg-Colsman, H. Waizy, Biodegradable magnesium-based screw clinically equivalent to titanium screw in hallux valgus surgery: short term results of the first prospective, randomized, controlled clinical pilot study, Biomedical Engineering Online, 12 (2013).

[3] J. Walker, S. Shadanbaz, T.B.F. Woodfield, M.P. Staiger, G.J. Dias, Magnesium biomaterials for orthopedic application: A review from a biological perspective, Journal of Biomedical Materials Research Part B-Applied Biomaterials, 102 (2014) 1316-1331.

[4] L.E. Claes, Mechanical characterization of biodegradable implants, Clinical materials, 10 (1992) 41-46. 
[5] T. Kraus, S.F. Fischerauer, A.C. Hanzi, P.J. Uggowitzer, J.F. Loffler, A.M. Weinberg, Magnesium alloys for temporary implants in osteosynthesis: in vivo studies of their degradation and interaction with bone, Acta Biomater, 8 (2012) 1230-1238.

[6] N.T. Kirkland, Magnesium biomaterials: past, present and future, Corrosion Engineering Science and Technology, 47 (2012) 322-328.

[7] J. Wang, J. Tang, P. Zhang, Y. Li, J. Wang, Y. Lai, L. Qin, Surface modification of magnesium alloys developed for bioabsorbable orthopedic implants: A general review, Journal of Biomedical Materials Research Part B-Applied Biomaterials, 100B (2012) 1691-1701.

[8] S. Hirano, K.T. Suzuki, Exposure, metabolism, and toxicity of rare earths and related compounds, Environmental Health Perspectives, 104 (1996) 85-95.

[9] C.K. Yuen, W.Y. Ip, Theoretical risk assessment of magnesium alloys as degradable biomedical implants, Acta Biomaterialia, 6 (2010) 1808-1812.

[10] S. Gogolewski, Bioresorbable polymers in trauma and bone surgery, Injury, 31 Suppl 4 (2000) 28-32.

[11] W.-D. Mueller, M.L. Nascimento, M. Fernandez Lorenzo de Mele, Critical discussion of the results from different corrosion studies of $\mathrm{Mg}$ and $\mathrm{Mg}$ alloys for biomaterial applications, Acta Biomaterialia, 6 (2010) 1749-1755.

[12] J.A. del Valle, O.A. Ruano, Superplasticity in a magnesium alloy prepared with bimodal grain size distributions developed by dynamic recrystallisation, Materials Letters, 62 (2008) 3391-3394.

[13] J.A. del Valle, F. Carreno, O.A. Ruano, Influence of texture and grain size on work hardening and ductility in magnesium-based alloys processed by ECAP and rolling, Acta Materialia, 54 (2006) 4247-4259.

[14] M. Alvarez-Lopez, M. Dolores Pereda, J.A. del Valle, M. Fernandez-Lorenzo, M.C. Garcia-Alonso, O.A. Ruano, M.L. Escudero, Corrosion behaviour of AZ31 magnesium alloy with different grain sizes in simulated biological fluids, Acta Biomaterialia, 6 (2010) 1763-1771.

[15] L. Choudhary, R.K.S. Raman, Magnesium alloys as body implants: Fracture mechanism under dynamic and static loadings in a physiological environment, Acta Biomaterialia, 8 (2012) 916-923.

[16] H. Wang, S. Guan, Y. Wang, H. Liu, H. Wang, L. Wang, C. Ren, S. Zhu, K. Chen, In vivo degradation behavior of Ca-deficient hydroxyapatite coated $\mathrm{Mg}-\mathrm{Zn}$ $\mathrm{Ca}$ alloy for bone implant application, Colloids and Surfaces B-Biointerfaces, 88 (2011) 254-259.

[17] D. Mushahary, C. Wen, J.M. Kumar, J. Lin, N. Harishankar, P. Hodgson, G. Pande, Y. Li, Collagen type-I leads to in vivo matrix mineralization and secondary stabilization of $\mathrm{Mg}-\mathrm{Zr}-\mathrm{Ca}$ alloy implants, Colloids and surfaces. B, Biointerfaces, 122 (2014) 719-728.

[18] Y.K. Pan, C.Z. Chen, D.G. Wang, T.G. Zhao, Improvement of corrosion and biological properties of microarc oxidized coatings on $\mathrm{Mg}-\mathrm{Zn}-\mathrm{Zr}$ alloy by optimizing negative power density parameters, Colloids and Surfaces BBiointerfaces, 113 (2014) 421-428.

[19] A. Abdal-hay, N.A.M. Barakat, J.K. Lim, Hydroxyapatite-doped poly(lactic acid) porous film coating for enhanced bioactivity and corrosion behavior of AZ31 Mg alloy for orthopedic applications, Ceramics International, 39 (2013) 183-195.

[20] M. Carboneras, L.S. Hernandez, J.A. del Valle, M.C. Garcia-Alonso, M.L. Escudero, Corrosion protection of different environmentally friendly coatings on 
powder metallurgy magnesium, Journal of Alloys and Compounds, 496 (2010) 442-448.

[21] C.A. Grillo, F. Alvarez, M.A.F. Lorenzo de Mele, Biological effects of magnesium particles degradation on UMR-106 cell line: Influence of fluoride treatments, Colloids and Surfaces B-Biointerfaces, 88 (2011) 471-476.

[22] S.F. Fischerauer, T. Kraus, X. Wu, S. Tangl, E. Sorantin, A.C. Hanzi, J.F. Loffler, P.J. Uggowitzer, A.M. Weinberg, In vivo degradation performance of micro-arcoxidized magnesium implants: a micro-CT study in rats, Acta Biomater, 9 (2013) 5411-5420.

[23] P.A. Yushkevich, J. Piven, H.C. Hazlett, R.G. Smith, S. Ho, J.C. Gee, G. Gerig, User-guided 3D active contour segmentation of anatomical structures: Significantly improved efficiency and reliability, Neuroimage, 31 (2006) 11161128.

[24] K. Donath, G. Breuner, A method for the study of undecalcified bones and teeth with attached soft-tissues - the sage-schliff (sawing and grinding) technique, Journal of Oral Pathology \& Medicine, 11 (1982) 318-326.

[25] D.F. Williams, On the mechanisms of biocompatibility, Biomaterials, 29 (2008) 2941-2953.

[26] H. Waizy, J.-M. Seitz, J. Reifenrath, A. Weizbauer, F.-W. Bach, A. MeyerLindenberg, B. Denkena, H. Windhagen, Biodegradable magnesium implants for orthopedic applications, Journal of Materials Science, 48 (2013) 39-50.

[27] C. Janning, E. Willbold, C. Vogt, J. Nellesen, A. Meyer-Lindenberg, H. Windhagen, F. Thorey, F. Witte, Magnesium hydroxide temporarily enhancing osteoblast activity and decreasing the osteoclast number in peri-implant bone remodelling, Acta Biomaterialia, 6 (2010) 1861-1868.

[28] R. Schmidhammer, S. Zandieh, R. Mittermayr, L.E. Pelinka, M. Leixnering, R. Hopf, A. Kroepfl, H. Redl, Assessment of bone union/nonunion in an experimental model using microcomputed technology, Journal of Trauma-Injury Infection and Critical Care, 61 (2006) 199-205.

[29] D.W. Buck, II, G.A. Dumanian, Bone Biology and Physiology: Part I. The Fundamentals, Plastic and Reconstructive Surgery, 129 (2012) 1314-1320.

[30] D.W. Buck, II, G.A. Dumanian, Bone Biology and Physiology: Part II. Clinical Correlates, Plastic and Reconstructive Surgery, 129 (2012) 950-956.

[31] K. Yu, L. Chen, J. Zhao, R. Wang, Y. Dai, Q. Huang, In vivo biocompatibility and biodegradation of a $\mathrm{Mg}-15 \% \mathrm{Ca}-3(\mathrm{PO} 4)(2)$ composite as an implant material, Materials Letters, 98 (2013) 22-25.

[32] D.-T. Chou, D. Hong, P. Saha, J. Ferrero, B. Lee, Z. Tan, Z. Dong, P.N. Kumta, In vitro and in vivo corrosion, cytocompatibility and mechanical properties of biodegradable $\mathrm{Mg}-\mathrm{Y}-\mathrm{Ca}-\mathrm{Zr}$ alloys as implant materials, Acta Biomaterialia, 9 (2013) 8518-8533.

[33] R.-G. Guan, I. Johnson, T. Cui, T. Zhao, Z.-Y. Zhao, X. Li, H. Liu, Electrodeposition of hydroxyapatite coating on $\mathrm{Mg}-4.0 \mathrm{Zn}-1.0 \mathrm{Ca}-0.6 \mathrm{Zr}$ alloy and in vitro evaluation of degradation, hemolysis, and cytotoxicity, Journal of Biomedical Materials Research Part A, 100A (2012) 999-1015.

[34] L. Xu, E. Zhang, D. Yin, S. Zeng, K. Yang, In vitro corrosion behaviour of Mg alloys in a phosphate buffered solution for bone implant application, Journal of Materials Science-Materials in Medicine, 19 (2008) 1017-1025.

[35] F. Witte, J. Fischer, J. Nellesen, H.A. Crostack, V. Kaese, A. Pisch, F. Beckmann, $\mathrm{H}$. Windhagen, In vitro and in vivo corrosion measurements of magnesium alloys, Biomaterials, 27 (2006) 1013-1018. 
[36] X. Gu, Y. Zheng, Y. Cheng, S. Zhong, T. Xi, In vitro corrosion and biocompatibility of binary magnesium alloys, Biomaterials, 30 (2009) 484-498.

[37] M.P. Staiger, A.M. Pietak, J. Huadmai, G. Dias, Magnesium and its alloys as orthopedic biomaterials: A review, Biomaterials, 27 (2006) 1728-1734.

[38] F. Witte, N. Hort, C. Vogt, S. Cohen, K.U. Kainer, R. Willumeit, F. Feyerabend, Degradable biomaterials based on magnesium corrosion, Current Opinion in Solid State \& Materials Science, 12 (2008) 63-72.

[39] F. Witte, The history of biodegradable magnesium implants: A review, Acta Biomaterialia, 6 (2010) 1680-1692.

[40] W. Wu, S. Chen, D. Gastaldi, L. Petrini, D. Mantovani, K. Yang, L. Tan, F. Migliavacca, Experimental data confirm numerical modeling of the degradation process of magnesium alloys stents, Acta Biomaterialia, 9 (2013) 8730-8739.

[41] D. Mantovani, F. Witte, The Thermec'2009 Biodegradable Metals, Acta Biomaterialia, 6 (2010) 1679-1679.

[42] E. Payr, Beitrage zur Technik der Blutgefass und Nervennaht nebst Mittheilungen uber die Verwendung eines resorbirbaren Metalles in der Chirurgie., Arch Klin Chir, 62 (1900) 67-93.

[43] E.D. McBride, Magnesium screw and nail transfixion in fractures, South. Madical J. , 31 (1938) 508-515.

[44] J. Kuhlmann, I. Bartsch, E. Willbold, S. Schuchardt, O. Holz, N. Hort, D. Hoeche, W.R. Heineman, F. Witte, Fast escape of hydrogen from gas cavities around corroding magnesium implants, Acta Biomaterialia, 9 (2013) 8714-8721.

[45] L.C. Gerstenfeld, Y.M. Alkhiary, E.A. Krall, F.H. Nicholls, S.N. Stapleton, J.L. Fitch, M. Bauer, R. Kayal, D.T. Graves, K.J. Jepsen, T.A. Einhorn, Threedimensional reconstruction of fracture callus morphogenesis, Journal of Histochemistry \& Cytochemistry, 54 (2006) 1215-1228.

[46] J.M. Garcia-Aznar, J.H. Kuiper, M.J. Gomez-Benito, M. Doblare, J.B. Richardson, Computational simulation of fracture healing: Influence of interfragmentary movement on the callus growth, Journal of Biomechanics, 40 (2007) 1467-1476.

[47] F.T. Beil, F. Barvencik, M. Gebauer, B. Beil, P. Pogoda, J.M. Rueger, A. Ignatius, T. Schinke, M. Amling, Effects of Increased Bone Formation on Fracture Healing in Mice, Journal of Trauma-Injury Infection and Critical Care, 70 (2011) 857-862.

[48] C. Kratzel, C. Bergmann, G. Duda, S. Greiner, G. Schmidmaier, B. Wildemann, Characterization of a rat osteotomy model with impaired healing, Bmc Musculoskeletal Disorders, 9 (2008).

[49] J. Wolff, Das Gesetz der Transformation der Knochen., Berlin, 1892.

[50] R. Huiskes, If bone is the answer, then what is the question?, Journal of Anatomy, 197 (2000) 145-156.

[51] E. Willbold, F. Witte, Histology and research at the hard tissue-implant interface using Technovit 9100 New embedding technique, Acta Biomaterialia, 6 (2010) 4447-4455.

[52] C. Castellani, R.A. Lindtner, P. Hausbrandt, E. Tschegg, S.E. Stanzl-Tschegg, G. Zanoni, S. Beck, A.M. Weinberg, Bone-implant interface strength and osseointegration: Biodegradable magnesium alloy versus standard titanium control, Acta Biomater, 7 (2011) 432-440.

[53] N. Erdmann, N. Angrisani, J. Reifenrath, A. Lucas, F. Thorey, D. Bormann, A. Meyer-Lindenberg, Biomechanical testing and degradation analysis of $\mathrm{MgCa} 0.8$ 
alloy screws: A comparative in vivo study in rabbits, Acta Biomaterialia, 7 (2011) 1421-1428.

[54] J. Kubasek, D. Vojtech, J. Lipov, T. Ruml, Structure, mechanical properties, corrosion behavior and cytotoxicity of biodegradable $\mathrm{Mg}-\mathrm{X}(\mathrm{X}=\mathrm{Sn}, \mathrm{Ga}, \mathrm{In})$ alloys, Materials Science \& Engineering C-Materials for Biological Applications, 33 (2013) 2421-2432.

[55] M. Carboneras, M.C. Garcia-Alonso, M.L. Escudero, Biodegradation kinetics of modified magnesium-based materials in cell culture medium, Corrosion Science, 53 (2011) 1433-1439.

[56] R.M. Lozano, B.T. Perez-Maceda, M. Carboneras, E. Onofre-Bustamante, M.C. Garcia-Alonso, M.L. Escudero, Response of MC3T3-E1 osteoblasts, L929 fibroblasts, and J774 macrophages to fluoride surface-modified AZ31 magnesium alloy, Journal of biomedical materials research. Part A, 101 (2013) 2753-2762.

[57] M. Thomann, C. Krause, N. Angrisani, D. Bormann, T. Hassel, H. Windhagen, A. Meyer-Lindenberg, Influence of a magnesium-fluoride coating of magnesiumbased implants (MgCa0.8) on degradation in a rabbit model, Journal of Biomedical Materials Research Part A, 93A (2010) 1609-1619.

[58] M. Carboneras, C. Iglesias, B.T. Perez-Maceda, J.A. del Valle, M.C. GarciaAlonso, M.A. Alobera, C. Clemente, J.C. Rubio, M.L. Escudero, R.M. Lozano, Corrosion behaviour and in vitro/in vivo biocompatibility of surface-modified AZ31 alloy, Revista De Metalurgia, 47 (2011) 212-223.

[59] E. Willbold, A.A. Kaya, R.A. Kaya, F. Beckmann, F. Witte, Corrosion of magnesium alloy AZ31 screws is dependent on the implantation site, Materials Science and Engineering B-Advanced Functional Solid-State Materials, 176 (2011) 1835-1840.

[60] R. Montoya, C. Iglesias, M.L. Escudero, M.C. Garcia-Alonso, Modeling in vivo corrosion of AZ31 as temporary biodegradable implants. Experimental validation in rats, Materials Science \& Engineering C-Materials for Biological Applications, 41 (2014) 127-133.

[61] R.B. Bell, C.S. Kindsfater, The use of biodegradable plates and screws to stabilize facial fractures, Journal of Oral and Maxillofacial Surgery, 64 (2006) 3139. 